\title{
Den Kopf über Wasser halten
}

\section{Ayurvedische Lebensführung bei Stress und Belastungsfaktoren}

AutorInnen: Kalyani Nagersheth, Martin Mittwede

\section{ZUSAMMENFASSUNG}

In Stresssituationen kommen bei Menschen laut Ayurveda die Eigenschaften stärker zum Vorschein, die den vorherrschenden Doshas entsprechen. Der Ayurveda kennt bei Stress und Stresssymptomen sowohl konstitutionstypische als auch allgemeine Maßnahmen der Therapie und Prophylaxe. Der zentrale Punkt der ayurvedischen Tagesroutine ist die Selbsteinölung. Sie wirkt unter anderem stärkend, schützend und entgiftend.

\section{Schlüsselwörter}

Ayurveda, Lebensführung, Konstitution, Stress, Atemtherapie, Ernährung, Selbsteinölung, Meditation, Achtsamkeit

\section{ABSTRACT}

According to Ayurveda, in stressful situations, the qualities that correspond to the predominant doshas become more prominent in people. Ayurveda knows constitution-typical as well as general measures of therapy and prophylaxis for stress and stress symptoms. The central aspect of the Ayurvedic daily routine is self-oiling. Among other things, it has a strengthening, protective, and detoxifying effect.

\section{Keywords}

Ayurveda, lifestyle, constitution, stress, breathing therapy, nutrition, self-oiling, meditation, attentiveness

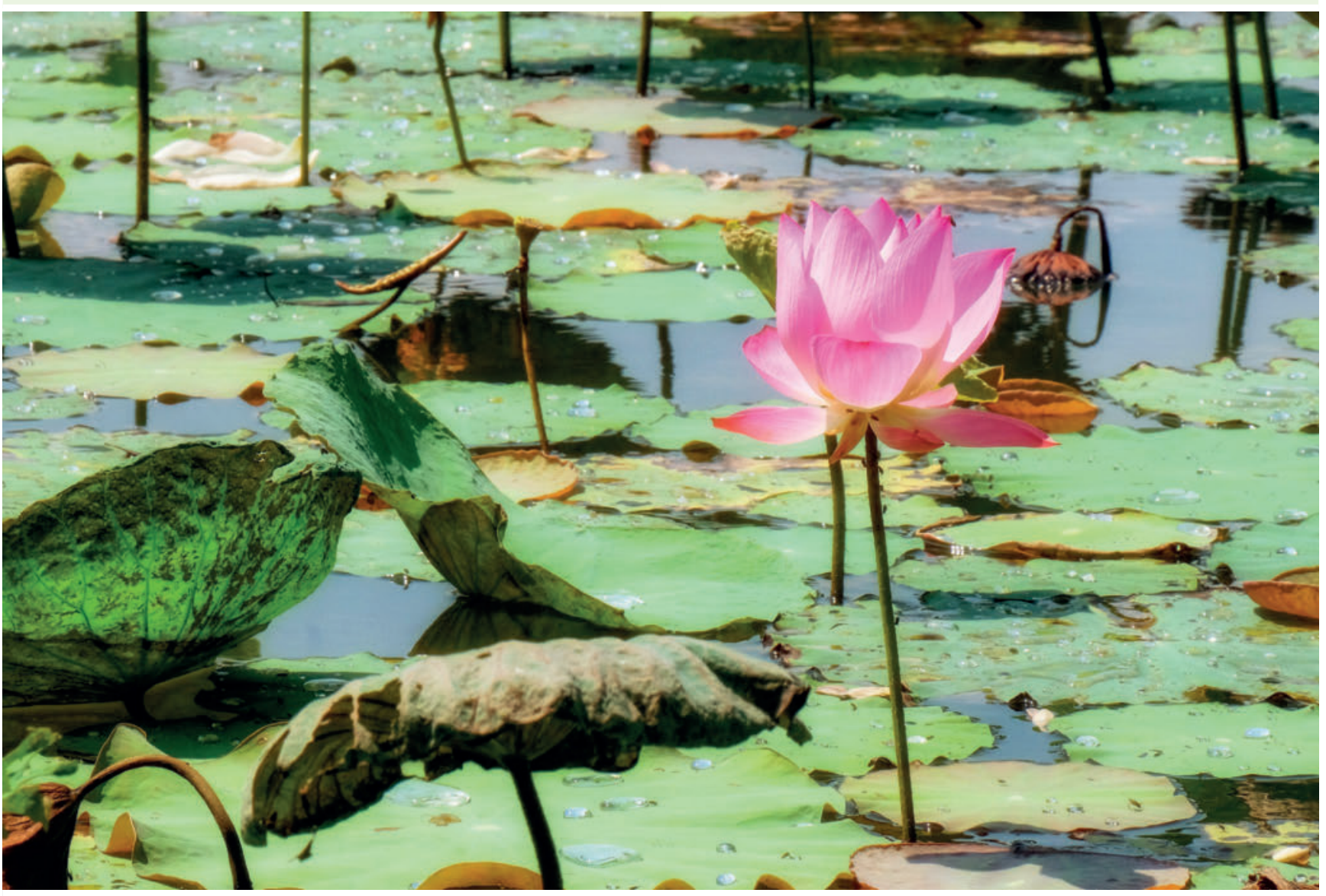

Abb. 1 Im Ayurveda wird die Lebensweise der Lotusblume als Metapher für eine meditative Geisteshaltung gesehen. Quelle: Kirsten Oborny/ Thieme Gruppe 
Aus ayurvedischer Sicht entsteht Stress, wenn jemand nicht entsprechend seiner Konstitution lebt oder zu viele Belastungsfaktoren auf ihn einwirken. Das wichtigste therapeutische Instrument bei Stress ist die ayurvedische Tagesroutine, eine individuelle Vorgehensweise bei der Behandlung ist das $\mathrm{A}$ und $\mathrm{O}$.

\section{Doshas unter Druck - Stress und Konstitution}

Im Ayurveda gibt es drei Doshas - die den Konstitutionstyp bestimmenden Prinzipien: Vata, Pitta und Kapha. Jeder Mensch hat Anteile aus allen dreien. Meist ist aber eines dominant. Stress verstärkt den jeweiligen konstitutionsbestimmenden Dosha. Das bedeutet, dass wir durch den Umgang des Patienten mit Stress Hinweise erhalten, welche Konstitution vorliegt. Wie sieht das für die einzelnen Doshas aus?

\section{Vata: nervös und sensibel}

Ein Mensch mit Vata-Betonung (Elemente Luft und Äther) wird durch Stress noch mehr zu Luft: Er verliert die Bodenhaftung und fühlt nur noch Leere im Körper. In Stresssituationen wird er das Chaos verstärken. Er gerät schnell in Stress, und Situationen, bei denen andere Menschen noch gelassen bleiben, stressen ihn bereits. Nicht selten tritt eine Hypersensibilität auf.

Typischerweise reagiert der Vata-Mensch emotional mit Furcht, Ängstlichkeit, Nervosität, Unruhe, Unsicherheit. Hinzu kommen körperliche Symptome wie Verstopfung, Herzklopfen, Atembeschwerden (es bleibt ihm die Luft weg).

\section{Pitta: Aktivität, bis nichts mehr geht}

Ein Mensch mit Pitta-Betonung (Element Feuer und etwas Wasser) verbrennt durch Stress (Stichwort Burnout-Syndrom), sein Feuer steigt, er entwickelt immer mehr Aktivität, bis plötzlich nichts mehr geht. Normalerweise ist ein Pitta-Mensch gut strukturiert und organisiert. Bei Stress versucht er, alles abzuarbeiten, zu funktionieren und übergeht dabei seine eigenen Bedürfnisse (Essen, Schlafen, Pause machen).

Emotional entwickelt er bei Stress vorzugsweise Irritation, Wut, Hass, Kritik, Neid, Eifersucht. Man kann seine Haltung unter Druck gut wie folgt zusammenfassen: „Die anderen sind an meinem Stress schuld, ich habe doch alles richtig gemacht." Auf der körperlichen Ebene entstehen Symptome der Übersäuerung, Durchfall, Herzbrennen, Hautausschläge oder Herpes.

\section{Kapha: schwer werden, schwer loslassen}

Ein Mensch mit Kapha-Betonung (Element Erde und Wasser) wird immer schwerer, er verfällt in Lethargie. Je mehr Stress vorherrscht, umso bewegungsloser wird er. Man merkt einem Kapha-Menschen den Stress nicht sofort an, aber er zeigt sich durch emotionales Essen, welches dann zu Übergewicht führt. Der Kapha-Mensch kann schwer loslassen, daher fällt es ihm schwer, sich selbst aus einer stressigen Situation hinauszubegeben.

Emotional entstehen Depression, Trauer und ein Gefühl der Wertlosigkeit. Körperlich zeigen sich Symptome der Stagnation wie Verstopfung und Übergewicht.

\section{Eine Konstitutionsbestimmung sollte möglichst schon im gesunden Zustand erfolgen. Häufig lassen sich dann stressbedingte Symptome durch eine konstitutionsbedingte Lebensweise vermeiden.}

\section{Konstitution als Kompass für die Lebensführung}

In der Realität gibt es natürlich neben den reinen Konstitutionstypen die Mischtypen, das heißt, es liegen Symptome aus zwei oder allen drei Doshas vor. Hier gilt es bei der Therapie Prioritäten zu setzen, um die Lebensqualität durch das Reduzieren der besonders belastenden Symptome zu verbessern. Wenn die Konstitution bereits bekannt ist, kann dem Stress vorgebeugt werden, indem die Ernährung, Lebensführung, manuelle Therapie, Heilpflanzen und auch Reinigungsmaßnahmen auf die Konstitution beziehungsweise die vorherrschenden Störungen abgestimmt werden.

Im Ayurveda werden auch Qualitäten der Konstitutionen erwähnt, welche die Berufswahl erleichtern können. So ist Vata sehr kreativ, Pitta ein Organisationstalent und Kapha ist ordentlich und zuverlässig. Durch den passenden Beruf können Stress und Druck vermieden werden. In der ayurvedischen Lehre vergleicht man einen Menschen, der seine Konstitution überfordernde Aktivitäten auf sich nimmt, mit einem Löwen, der versucht, einen Elefanten zu erbeuten und wegzuschleifen. Man sollte sich seiner Konstitution entsprechend verhalten, die eigenen Ressourcen richtig einschätzen und eher die konstruktiven Qualitäten ausleben, als sich zu überfordern.

\section{Vata: braucht Halt und Fülle}

Wichtig für den Vata-Menschen ist ein sehr regelmäßiges Leben (quasi nach Stundenplan): immer um die gleiche Uhrzeit aufstehen und schlafen gehen, immer um die gleiche Uhrzeit essen, regelmäßige Arbeitszeiten und Aktivitäten. Das wirkt den Qualitäten von Vata (leicht, trocken, kalt, rau, beweglich, klar) entgegen und gibt Stabilität. Besonders die Qualität Beweglichkeit wird durch Regelmäßigkeit reduziert. 
Die Ernährung sollte warm, schwer (erdend, nährend), süß und feucht (wirkt der Qualität „trocken“ entgegen) sein, dazu zählen zum Beispiel Suppen, Getreidebreie, Gemüse mit Sahnesoßen. Rohkost (zum Beispiel Salat) und trockene Nahrungsmittel (zum Beispiel Knäckebrot) sollten vermieden werden, denn sie verstärken Vata-Eigenschaften.

Für Vata-Menschen sind alle ayurvedischen Ölbehandlungen (Massagen [Abhyanga]) geeignet, denn Öl wirkt der Eigenschaft Trockenheit entgegen.

\section{Pitta: muss sein Feuer kontrollieren}

Da beim Pitta-Menschen das Element Feuer überwiegt, sollte er sich nicht zu viel Hitze aussetzen (Sauna) und keine scharfe Nahrung zu sich nehmen. Um die Energie abzubauen, eignet sich Sport zum Ausgleich sehr gut. In Stresssituationen sollten Pitta-Menschen lernen zu delegieren und Dinge abzugeben.

Regelmäßig und in ausreichender Menge eingenommene Nahrung mit vielen grünen und gelben Anteilen (Bitterstoffen) ist empfehlenswert, zum Beispiel grüne Blattsalate, grüne Gemüse, grüne Küchenkräuter, Kurkuma. Ayurvedische Fußmassagen mit Ghee (geklärter Butter) erden Pitta besonders. All diese Maßnahmen reduzieren die Pitta-Eigenschaften: heiß, flüssig, fließend, sauer, leicht ölig, chilischarf und messerscharf.

\section{Kapha: braucht etwas mehr Leichtigkeit}

Für Kapha-Menschen sind leichte, aber warme Nahrung und viele scharfe Gewürze zu bevorzugen. Kapha und die entsprechenden Qualitäten (schwer, stabil, ölig, süß, feucht, kalt) werden durch scharfe, bittere und zusammenziehende Nahrungsmittel reduziert. Kapha-Menschen können auch Mahlzeiten auslassen und sollten unbedingt auf Zwischenmahlzeiten verzichten, dies fördert die Leichtigkeit und wirkt der schweren Eigenschaft entgegen.

Kapha-Menschen lieben die Routine und fühlen sich durch Veränderungen sofort gestresst. Daher brauchen sie Freunde oder Kollegen, die ihnen den Übergang bei Veränderungen erleichtern. Kapha-Typen dürfen etwas mehr Neugier entwickeln und immer mal etwas Neues ausprobieren. Besonders die anregenden ayurvedischen Massagen (Kräutermassagen, Trockenmassagen) sind für sie geeignet.

\section{Bala und sattva überfordert - Stress und Belastungsfaktoren}

Neben der Konstitution betrachtet der Ayurveda sehr genau die Einflüsse, die auf einen Menschen einwirken. Eine Dysbalance entsteht immer dann, wenn regelmäßig - das heißt zu häufig - Belastungen auftreten, die die körperliche Kraft (bala) und die psychische Stabilität (sattva) überfordern. Aus ayurvedischer Sicht darf ein gesunder Mensch auch schon mal über seine Kapazitäten hi- nausgehend gefordert sein. Wenn es aber zu Dauerbelastungen wie täglichen Überstunden oder Streitigkeiten mit dem Partner oder zu intensiven Belastungsreizen kommt, wird das System nachhaltig gestört. Kraft und psychische Stabilität nehmen ab (Missverhältnis zwischen Ressourcen und Anforderungen). Dies endet in Erschöpfung.

Im schlimmsten Fall verlieren dann Menschen die Fähigkeit, zwischen dem zu unterscheiden, was sie belastet und dem, was sie stärkt. In diesem gestörten Zustand dreht der Betroffene dann die Bewertung um, das heißt, er hält das Wohltuende für belastend und das Belastende für wohltuend. Das gilt dann auch für die Empfehlungen des Therapeuten, die nicht als stärkend und hilfreich empfunden werden. Zum Beispiel arbeitet dann ein Patient weiter, obwohl er längst schlafen müsste, oder er raucht, obwohl er dringend damit aufhören müsste. Er nimmt nämlich an, dass die Zigarette oder das Weiterarbeiten hilft, um gegen die Erschöpfung anzukommen.

Hier bedarf es einer intensiveren Arbeit, um einem Menschen das natürliche Empfinden für seinen Körper und dessen Bedürfnisse wieder zu vermitteln. Der Patient muss lernen, die eigenen Bedürfnisse wahrzunehmen und nicht einfach zu funktionieren. Die Arbeit besteht darin, dem Patienten Fragen zu stellen und ihn dadurch zu einem bewussten In-sich-Hineinhören zu bringen.

\section{Anamnese, Atem und Achtsamkeit - Wege aus dem Stress}

Schon die Anamnese ist Therapie. Der Patient muss unterscheiden, was er preisgibt und was nicht. Er muss den Mut haben, sich mitzuteilen. Durch Nachfragen, wie der Patient auf Stress reagiert, wird schon sein Bewusstsein gefördert. Im Ayurveda hilft die liebevolle Zuwendung dem Patienten, wieder mit seinen Emotionen in Kontakt zu kommen.

Die Atemtherapie ist im Ayurveda eine wichtige Säule, denn der Atem stellt die Verbindung vom Körper zur Psyche dar. Diese Verbindung läuft über Pranavata. Pranavata ist für den Atem zuständig. Daher können über Atemübungen der Geist/die Psyche beeinflusst/beruhigt werden. Im Yoga gibt es Pranayama-Atemübungen. Auch diese können der Konstitution angepasst werden. Im Ayurveda wird empfohlen, die Ausatemphase zu verlängern: zum Beispiel beim Einatmen bis zwei zählen, beim Ausatmen bis vier zählen, möglichst in einer entspannten Haltung (im Liegen).

Zusätzlich ist auch die komplementäre Vorgehensweise sinnvoll: Bei starken psychischen Spannungen können beispielsweise ayurvedische Manualtherapien und Ölmassagen (Abhyanga) helfen, bei rein körperlichen Beschwerden lohnt es sich, auch auf die Stärkung des psychischen Gleichgewichts zu schauen. 


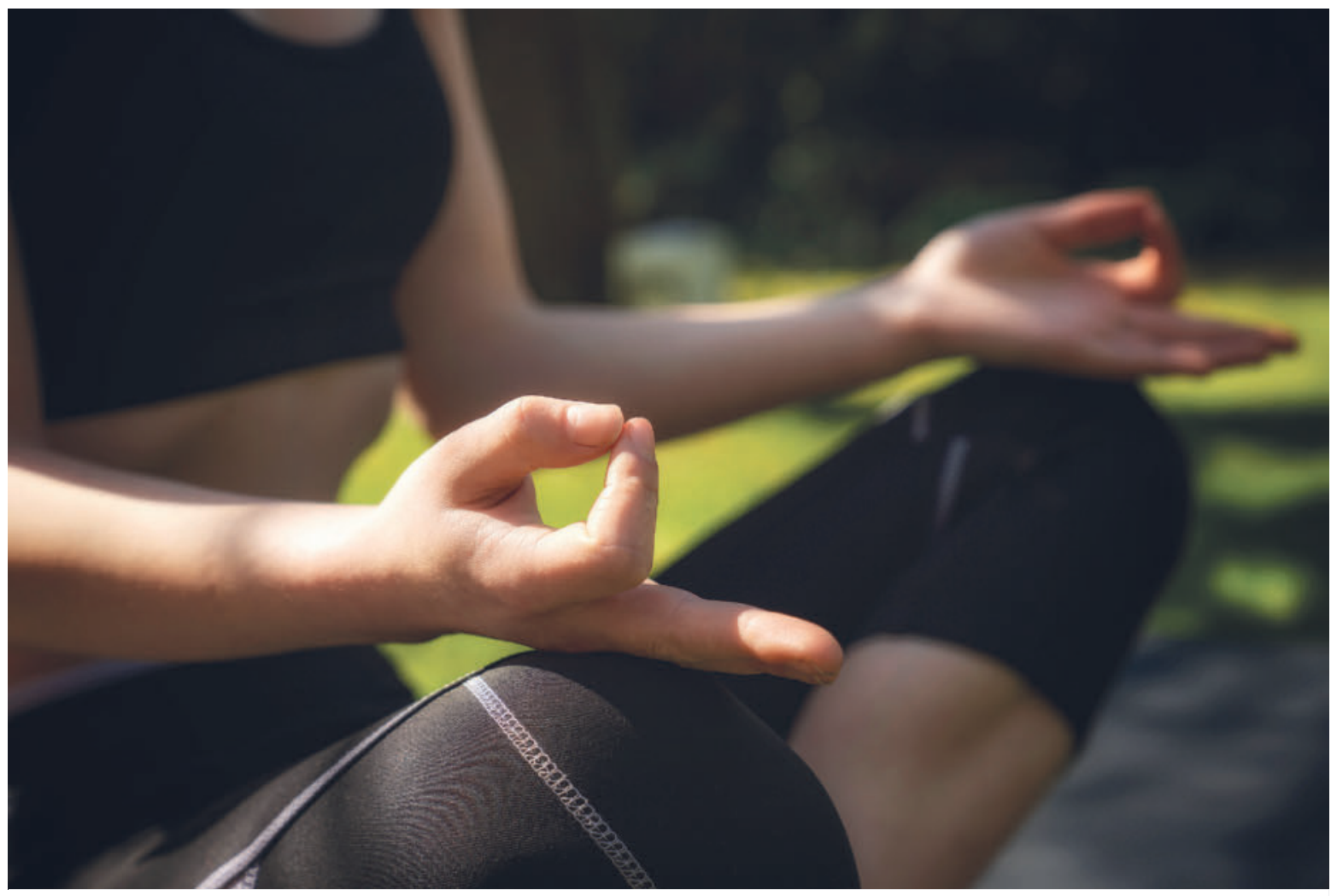

Abb. 2 Tägliche Meditation und eine meditative Grundhaltung sind auch im Ayurveda wichtige Werkzeuge, um mit Stressoren umzugehen und unerwünschten Stressreaktionen vorzubeugen. Quelle: Kirsten Oborny/Thieme Gruppe

\section{Der Lotus als Vorbild - Achtsamkeit im Sinne des Ayurveda}

Tägliche Meditation oder eine meditative Grundhaltung bilden die ayurvedische Entsprechung für das inzwischen moderne Achtsamkeitstraining. Nicht funktionieren, sondern Dinge bewusst tun beziehungsweise bewusst wahrnehmen ist eine lebenslange Übung - und hilft, Automatismen zu vermeiden. Hier eignet sich unter anderem die aus dem Ayurveda bekannte Metapher der Lotusblume. Sie wird aus dem Wasser geboren, blüht jedoch über der Wasseroberfläche. Das Wasser perlt an den Lotusblättern ab. Das bedeutet, dass sich die Lotuspflanze nicht direkt mit dem sie umgebenden Element verbindet und dadurch nicht durch das (schmutzige) Wasser (negativ) beeinflusst wird. Die Lotusblume verweilt in einer quasi meditativen Haltung.

Doch beachten Sie: Gerade für Menschen, die sehr gestresst sind, kann eine zusätzliche tägliche Meditationszeit den Stress subjektiv verstärken. Für solche Menschen ist das Üben der Achtsamkeit im Alltag sehr hilfreich, denn sie verbraucht keine zusätzliche Zeit und erzeugt keinen Leistungsdruck: Achtsamkeit in der Warteschlange im Supermarkt, Achtsamkeit im Autostau, Achtsamkeit beim Blumengießen oder - ayurvedisch sehr wichtig - Achtsamkeit beim Kochen können hier hilfreich sein. Achtsamkeit und eine meditative Grundhaltung bedeuten unter ande- rem, dass man nicht fünf Sachen gleichzeitig erledigt, sondern alle Aktionen bewusst wahrnimmt. Beim Kochen zum Beispiel sollte man sich vorher bewusst sein, auf welche Nahrungsmittel man ein Bedürfnis verspürt (immer unterscheiden, ob es ein Bedürfnis, eine Gewohnheit oder Lust ist), und sich beim Kochen bewusst machen, dass man sich etwas Gutes tut und den Körper und den Geist nährt (sich Liebe zuführt).

\section{Wieder differenziert wahrnehmen}

Im Stresszustand neigen viele Menschen zu einem Schwarz-Weiß-Denken. Häufig suchen sie dann nach der einen Lösung, die den Stress zum Verschwinden bringen soll. Aus ayurvedischer Sicht hängen alle Lebensfaktoren miteinander zusammen. Wenn wir von 10 Belastungsthemen bei 3 oder 4 eine gefühlte Reduktion um 10 oder $20 \%$ vornehmen, kann dies schon ausreichen, damit sich das System wieder ins Gleichgewicht einpendelt. Wenn man dann achtsam an der Reduktion von Belastungen weiterarbeitet, kann dies nachhaltig dem Stress den Nährboden entziehen.

\section{Der Königsweg - die Tagesroutine}

Das vermutlich wichtigste Instrument bei Stress ist die ayurvedische Tagesroutine. Sie sollte bereits im gesunden Zustand eingeübt werden, damit sie in stressigen Phasen 


\section{AUS DER PATIENTENAKTE}

\section{Wieder mehr nach der Konstitution leben}

Eine 36-jährige Patientin, $61 \mathrm{~kg}$ bei $173 \mathrm{~cm}$, kam mit unklaren Beschwerden, die eventuell zu einem Reizdarmsyndrom passen könnten (Durchfallattacken). Die Magen-Darm-Spiegelung zeigte keinen Befund. Schon als Jugendliche hatte sie immer das Gefühl, einen Stein im Magen zu haben. Zusätzlich bestanden Kopf- und Rückenschmerzen, sodass sie immer wieder zu Ibuprofen greifen musste.

Sie wurde per Kaiserschnitt entbunden, da sie falsch herum lag. Als Kleinkind litt sie unter Neurodermitis. Als Kind musste sie oft Antibiotika einnehmen wegen Erkrankungen im HNO-Bereich. Die Erziehung war streng, sie war ein ruhiges, angepasstes Kind. Nach dem Studium (Sport und Pädagogik) hat sie heute eine leitende Tätigkeit, Vollzeit mit vielen Überstunden, bei der sie viel organisieren muss und sehr selbstständig entscheiden darf. Auf ihre Ernährung legt sie keinen größeren Wert und isst so nebenher. Für ihren eigentlich geliebten Sport nimmt sie sich keine Zeit. Emotionen (besonders Wut) erlaubt sie sich nicht.

Die ayurvedische Diagnose zeigt zwar eine Pitta-Konstitution, das Pitta wird aber nicht ausreichend ausgelebt. Meist stammt dies bereits aus der Kindheit, wenn durch die Erziehung die Konstitution unterdrückt wird.

Es erfolgte eine ausführliche Aufklärung über die positiven Eigenschaften von Pitta. Insbesondere Sport wurde empfohlen. Dabei auch Kampfsport, um die unterdrückte Wut sozial verträglich auszuleben. Sowohl stoffwechselanregende Massagen als auch Stirngüsse wurden durchgeführt. Die Patientin setzte viele Punkte der Tagesroutine um. Die Ernährung war Vata-reduzierend. Durch die Reduktion von Vata konnte sich Pitta wieder frei entfalten (Vata blockierte Pitta nicht mehr). Nach zwei Monaten besserten sich auch die Verdauungsbeschwerden, vermutlich weil die Patientin sich mehr in ihrer Konstitution fühlte und sich dadurch selbst etwas Druck nahm.

bereits als Routine besteht. Disziplin (Routine) kann besonders in schwierigen Lebenssituationen Halt und Stabilität geben. Diese Tagesroutine darf grundsätzlich täglich durchgeführt werden, sollte aber zumindest dreimal pro Woche erfolgen, erst dann wird sie zur Gewohnheit.

Zu bestimmten Tageszeiten herrschen bestimmte Doshas vor. Daher sollten die verschiedenen Konstitutionen zu bestimmten Tageszeiten aufstehen: Kapha vor Sonnenaufgang, Pitta mit Sonnenaufgang, Vata nach Sonnenaufgang.

Nach dem Aufstehen sollte jeder ein bis zwei Gläser warmes bis heißes Wasser trinken. Dies spült den Verdauungskanal und weckt den Körper auf. Außerdem wird die Darmperistaltik angeregt. Dies führt zu einem regelmäßigen Stuhlgang und beugt Verstopfung vor.

Danach erst sollte die Zunge geschabt werden. Dies kann mit einem Zungenschaber oder mit einem Teelöffel ge- macht werden, nicht aber mit der Zahnbürste, da sich sonst der Belag in den Borsten sammelt. Durch das Schaben der Zunge wird die natürliche Entgiftung unterstützt. Über Nacht sammelt sich immer ein leichter Belag auf der Zunge. Der Körper entgiftet während der Nacht. Indem der Belag abgeschabt wird, werden belastende Stoffe aus dem Körper entfernt. Außerdem werden durch das Schaben die einzelnen Organe (auf der Zunge vertreten) aktiviert.

Als Nächstes ist in der ayurvedischen Tagesroutine Zeit für den Stuhlgang. Selbst wenn kein Stuhldrang vorliegt, sollte man einige Minuten sitzen, besser hocken. Beim Hocken geht es darum, die Lendenwirbelsäule zu entlordosieren und dadurch die letzte Darmschlinge ein wenig in Streckung zu bringen, dann kann der Stuhlgang besser rutschen. Zusätzlich hilft die Bauchpresse durch die Oberschenkel am Bauch, den Stuhl im Darm besser vorwärts zu bewegen (eine Art Darmmassage). Dies kann durch Gewohnheit zu einem regelmäßigen Stuhlgang führen. Dabei sollte man jedoch nicht pressen.

\section{Nun ist das Einölen an der Reihe}

Der zentrale Punkt der ayurvedischen Tagesroutine besteht in der Selbsteinölung. Dabei trägt man zum Beispiel warmes Sesamöl auf den ganzen Körper auf (ähnlich wie eine Bodylotion). Der indische Arzt Caraka schrieb: „So, wie die Achse eines Wagens durch Ölung stark und belastbar gemacht wird, wird der menschliche Körper durch Öl stark und bekommt eine weiche Haut. [...] Wenn jemand regelmäßig Ölmassagen erhält, wird der Körper selbst durch Verletzungen und anstrengende Arbeit nicht stark beeinträchtigt“ [1].

Laut Caraka wird der Körper durch Selbsteinölung auch für Vata-Erkrankungen wie Stress, innere Unruhe, Schlafstörungen, Trockenheit, Verstopfung, Arthrose oder Schmerzen weniger anfällig. Denn Vata ist im Tastsinn dominant - und dieser sitzt in der Haut. Die Selbsteinölung macht generell widerstandsfähig gegen Erschöpfung und Anstrengung und verlangsamt den Alterungsprozess [1].

Durch das Öl findet sowohl eine Entlastung (Entgiftung über die Haut) als auch eine Nährung statt. Außerdem bildet es - obwohl es später abgeduscht wird - eine Schutzhülle für den ganzen Tag. Stress und Druck können ähnlich dem Lotuseffekt an dieser Schutzhülle abgleiten und dringen nicht in den Menschen ein.

Das Öl sollte einige Zeit auf der Haut bleiben, daher empfiehlt es sich, nach der Selbsteinölung einen alten Jogginganzug anzuziehen. Während das Öl auf der Haut verbleibt, kann man konstitutionsentsprechende Yogaübungen machen, meditieren oder frühstücken. Dann wird das Öl abgeduscht, das ist wichtig, um die fettlöslichen Gifte, die aus dem Körper gezogen wurden, abzuwaschen. Dazu ist auch etwas Seife erforderlich. Unter der Dusche kann das 
Ölziehen erledigt werden: Den Mund ca. zu zwei Dritteln mit Öl füllen, das Öl für 10-15 Minuten im Mund hin und her bewegen, dann ausspucken. Dies reinigt die Mundhöhle. Zusätzlich kann Öl oder Ghee (geklärte Butter) in die Nase gegeben werden, um die Sinne zu stärken und für geistige Klarheit zu sorgen.

Interessenkonflikt

Die AutorInnen geben an, dass kein Interessenkonflikt besteht.

\section{Korrespondenzadressen}

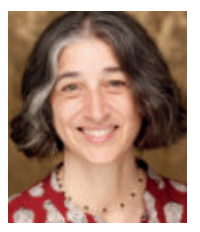

\section{Kalyani Nagersheth}

hat in Frankfurt ihr Medizinstudium absolviert. Im Jahr 2000 privatärztliche Praxis für ayurvedische Medizin in Frankfurt. Ab 2007 Wechsel nach Kassel, dort ab 2009 Leitung der ayurvedischen Abteilung der Habichtswald-Klinik. Seit 2013 Fachärztin für Physikalische und Rehabilitative Medizin, Zusatzbezeichnung Naturheilverfahren sowie ärztliche Psychotherapeutin. Seit 2013 wieder in Frankfurt in einer privatärztlichen Praxis sowie medizinische Leitung an der Europäischen Akademie für Ayurveda.

Dr. med. Kalyani Nagersheth

E-Mail: info@ayurveda-ffm.de

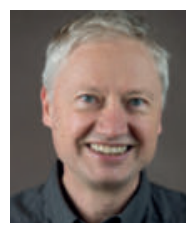

\section{Martin Mittwede}

studierte Indologie und Religionswissenschaften; Durchführung eines interdisziplinären Forschungsprojekts der DFG über AyurvedaMedizin; Habilitation an der Universität Frankfurt am Main. Er verfügt über langjährige Erfahrungen in der ärztlichen Fortbildung und entwickelte ein ernährungsmedizinisches Curriculum. Aktuell ist er als Studienleiter des Master-Studiengangs Ayurveda-Medizin an der Europäischen Akademie für Ayurveda und in eigener psychotherapeutischer Praxis tätig. Prof. Dr. Martin Mittwede E-Mail: martin.mittwede@ayurveda-akademie.org

\section{Zitierweise für diesen Artikel}

(c) DHZ 2020; 3: 14-19

Literatur

[1] Samhitā Caraka. Sūtra Sthāna 5: 85-93

Bibliografie

EHK 2021; 70: 96-101

DOI 10.1055/a-1395-4677

ISSN 0014-0082

(c) 2021. Thieme. All rights reserved.

Karl F. Haug Verlag in MVS Medizinverlage Stuttgart GmbH \& Co. KG, Oswald-Hesse-Straße 50, 70469 Stuttgart Germany 